\title{
Extractivismo marino-colonial. Apropiación asimétrica de recursos marinos en el golfo de California (México) siglos XVI-XXI
}

\author{
Antonio Ortega Santos*
}

\section{RESUMEN}

En la península y en el golfo de California, el proceso de conquista colonial impuso un proceso de antropización-colonización de la relación sociedad-naturaleza a lo largo del tiempo moderno y contemporáneo. Desde el siglo XVI, este cambio se implementó mediante la inserción de los recursos naturales terrestres y marinos disponibles en el territorio sudcaliforniano en el marco de la Economía Mundo. La península y el golfo de California se vieron sometidos a un proceso, a largo plazo, de saqueo y despojo territorial que denomino "Apropiación Asimétrica" (que se traduce en una línea temporal de cuatro momentos históricos, algunos coetáneos, desde el siglo XVI al siglo XXI). En el primer momento de esta línea temporal, se describe en el texto cómo la Corona transfirió, mediante diversos sistemas de cesión territorial, el control de recursos (perlas, acuacultura, guano, sal, etc.) hacia empresarios privados encargados de extraer el capital natural para comercializarlo en mercados globales entre el siglo XVI y el siglo XX. Esta capitalización/privatización de recursos costeros-marinos a lo largo del siglo XX se evidenció en un proceso de especulación financiero territorial, orientado al desarrollo del turismo de masas como eje del modelo de desarrollo económico (tercer y cuarto momento histórico de la Apropiación Asimétrica que se analiza en el texto.

La propuesta de metodología de investigación parte del trabajo que desarrollamos en el campo de la Historia Ambiental, entendida como estudio sobre las relaciones entre sociedad y naturaleza a lo largo del mundo moderno y contemporáneo, estudiado mediante el análisis de los documentos archivísticos y bibliografía de coetáneos, viajeros o documentos institucionales. Esta propuesta metodológica supone el estudio de los cambios territoriales, ambientales y socioeconómicos junto al impacto de los mismos en el capital natural del territorio sudcaliforniano, procesos que generan conflictos y luchas contra el extractivismo y despojo como modelo económico. Desde el campo de la Historia Ambiental, el concepto de extractivismo es definido como transferencia de bienes naturales de manejo comunitario hacia la esfera del mercado, enajenados los pueblos y beneficiados sectores empresariales con este proceso de privatización. De igual manera, el concepto de apropiación asimétrica implica la identificación de cómo la transferencia verificada mediante el extractivismo y despojo territorial impacta sobre la arquitectura del modelo de desarrollo económico generando episodios y realidades de injusticia ambiental, dando lugar a importantes desequilibrios territoriales. Estos desequilibrios territoriales tienen una matriz histórica que la Historia Ambiental permite narrar como un contínuum, pero que como práctica de colonización del territorio llega hasta inicios del siglo XXI como demuestra el estudio del impacto del capitalismo financiero en Baja California.

\section{Palabras clave}

Historia Ambiental; Extractivismo Marino-Costero;Apropiación Asimétrica; Baja California Sur; estudios decoloniales.

\section{TITLE}

Ocean Colonial Extractivism. Asymmetric Appropriation of Marine Resources in the Gulf of California, $\mathrm{XVI-XXI}$ centuries

\section{Extended Abstract}

Since the arrival of the Jesuits to the Baja California Sur Peninsula, the conquest and colonization of the territory involved an intense process of anthropization of the area.This process involved the insertion of natural resources within the framework

\section{DOI:}

https://doi.org//0.15366/relacionesinternacionales2021.46.006

Formato de citación recomendado:

ORTEGA SANTOS,Antonio (2021). "Extractivismo marino-colonial.Apropiación asimétrica de recursos marinos en el golfo de California (México) siglos XVI-XXI”, Relaciones Internacionales, n 46, pp. 99-II7.
*Antonio ORTEGA SANTOS, Profesor Titular de Historia Contemporánea de la Universidad de Granada. Investigador Líder del Grupo de Investigación HUM952 South Training Action Network of

Decoloniality.

Miembro de

la Ejecutiva de la Sociedad

Latinoamericana y Caribeña de Historia Ambiental (SOLCHA).

Contacto:aortegas@ ugr.es

Recibido: I5/09/2020

Aceptado: 28/0I/202। 
of the World Economy, the conversion of agricultural land under forms of missionary colonization, and, what is less well known, a process of commodification of the peninsula, initiating a cycle of extractivism of land and marine assets under state or private concession forms. If, in the first missionary phase a territory of manifest agricultural vocation was woven with the "architecture" of agroecosystems of orchards (called Oasis under a Eurocentric perspective) with the arrival of the Mexican Nation State -throughout the 19th century- the Peninsula and The Gulf were subjected to a process of looting that we come to call Asymmetric Appropriation. This accumulation by dispossession was implemented on a series of goods held in common by the inhabitants of the Peninsula and the Gulf, oriented to local or national businesses. However, since the mid-nineteenth century it would be inserted in the framework of global marketing circuits. As the first phase gave way to the arrival of new episodes of accumulation by dispossession during the "discovery" of the Baja California Peninsula as a land of promise for extractive fishing activity (pearls, aquaculture, etc.), it also gave rise to the last episode of gentrification of the territory through mass tourism. This last phase of the rupture allows us to speak of the Baja California Peninsula as an "Exceptional Space" in which high levels of looting of natural resources for global accumulation have been witnessed over two centuries. In this context, the Jesuit missions were installed as not only ways of semiotic and evangelizing conquest of the space and its inhabitants, but as true units of production and consumption. This moves towards a hybridization of Mediterranean garden crops, arriving at biotypes typical of the orchards and Mediterranean agroecosystems, together with existing plants.A first milestone of our work is to decipher and catalog the entry of these crops and the "creation" of new agricultural production spaces. Oriented to subsistence, these represent a productive optimum that extends their life for much of the 19th century and beyond the departure of the different religious orders- this is not attended to in this paper, but connected with the Europeanization of the agroecosystem in lower California.

The methodology and objectives stem from the perspective of environmental history of the GoC, from the I6th century to the present, and in order to identify the changing forms of perception and constants of asymmetric appropriation of the coastal marine space through the use and management of resources. As a general hypothesis, for future investigations, the GOC's biocultural wealth is threatened by an historical extractive regional economy. Only the territorial re-appropriation of the region by the inhabitants will allow for a movement towards local socio-ecological sustainability, reconsidering the production of its space through community appropriation of the marine-coastal territory based on the principles of sustainability. In this perspective, tools and methodologies have been used both from the field of archival documentary information (General Archive of the Nation, Pablo López Martínez Archive, La Paz, Baja California Sur, Travel Notebooks, Official Reports of Institutions) together with the enormous quantitative and qualitative value of the information obtained from other publications (official reports, travellers' books, etc.). With this sum of information from historical archival sources typical of Environmental History (either primary or secondary), together with the research matrix with a profile attended to socio-environmental changes, a narrative of the looting / pillaging of the Gulf of California has been structured with two case studies: the history of pearl extraction in the Gulf of California (which occurred in the first two periods of asymmetric appropriation and is explained in Section 2 of this article) and the impact study of the tourism model and gentrification of the territory in the coastal areas of the Peninsula thanks to international financial speculative pressure, especially since the global financial and economic crises of the last ten years (from 1980s until the present).

With these two examples it is possible to mark a historical matrix, a timeline of the historical process of plundering, of colonial appropriation of the Gulf of California, until today.This historical line allows us to assume that colonial practices have survived beyond the independence processes of the first third of the 19th century, until today. Coloniality comes hand in hand with the design of a program of oligopolistic appropriation of natural resources that has multiple episodes as evidenced in section 2 Table I. The dynamics of the conversion of this territory into a place of multiple extractivist economies is implemented (already addressed in other personal and collective publications) to satisfy the sociometabolic requirements of the World Economy; that is, of capitalism.

This proposal entails a necessary, albeit rarely seen, look at the processes of maritime extractivism with a colonial profile - emerging in European contexts in recent years - that break with the hegemony of territorial studies in an academic context in order to understand the coexistence between human societies and the environment. For socio-environmental studies, new lines of investigation are opened that must be supported by both quantitative -the design of tools on socioenvironmental metabolism in community fishing environments- and qualitative information -understanding that the conflicts and resistance to these economies of plunder are nothing more than evident phenomena of the new cycles of struggles for life, of struggles with a decolonial matrix-. The field of Environmental History thus plays as a meeting point, an ecology of knowledge and an interdisciplinary arena to create socially committed knowledge in the face of the ecological and civilizational crisis in which we are immersed. From the field of Environmental History, the concept of extractivism is defined as the transfer of natural assets from community management to the market sphere and the capitalization of nature. Similarly, the concept of asymmetric appropriation implies the identification of how the transfer carried out through extraction and territorial dispossession impacts on the architecture of the economic development model, generating episodes and realities of environmental injustice, and giving rise to important territorial imbalances. These territorial imbalances have a historical matrix, which Environmental History allows us to narrate as a continuum. Moreover, understood as a practice of colonizing the territory, this reaches the beginning of the 21 st century, as is seen in the study of the impact of financial capitalism in Baja California.

\section{KEYWORDS}

Environmental history; coastal-ocean extractivism; asymmetric appropriation; Lower California; decolonial studies. 
Aplicado al caso de estudio aquí propuesto, con la Historia Ambiental se pueden definir

los procesos históricos de apropiación asimétrica en el territorio, traducida en una línea temporal de capitalización de los recursos naturales, que despojaron a los pueblos originarios de los mismos, para mediante sistemas de concesión, facilitar que sectores empresariales pudieran extraer la biomasa marina o costera para su venta en mercados globales. Se apuesta por una aproximación a una historia ambiental de mares y océanos, con la que iniciar una línea de investigación sobre la dimensión cualitativa de los cambios en la interfase tierra-agua de zonas costeras, fruto de una "colonialidad de la naturaleza" que transita tanto durante el periodo colonial en sí, como en la contemporaneidad, en el marco legislativo de construcción de los EstadosNación postindependencia.

El presente artículo se organiza conceptualmente y epistemológicamente desde el campo de la Historia Ambiental, emergente en muchos lugares de enunciación en el campo latinoamericano y europeo en los últimos decenios, aunque tendiendo puentes de diálogo inter epistémicos con la Ecología Política. La Historia Ambiental no es solo una nueva especialidad de la historiografía contemporánea añadida al "desmigajamiento" de la postmodernidad. Se propone como una "revolución conceptual" alimentada desde una conciencia-ciudadanía global frente al impacto de los procesos de transformación humana en su relación con la naturaleza, alejándose de la especialización científica y la parcelación del conocimiento acaecida en los últimos decenios. Este discurso historiográfico nace de la toma de conciencia de la propia crisis ambiental que nos envuelve, del impacto del cambio climático, de la deforestación a escala mundial, tiene una serie de rasgos epistemológicos y ontológicos comunes, como pilares constructores del nuevo paradigma. Interdisciplinariedad frente a espíritu analítico, integridad frente a fragmentariedad, centralidad de lo relacional frente a sustancia del mecanicismo; importancia de la dimensión tiempo; dominación de perspectiva/ética biocéntrica, son notas de ruptura del discurso historiográfico hasta ahora dominante, en las que se asienta la fundamentación de la historia ambiental. Ni historizamos la ecología, ni ecologizamos la historia. Proponemos una visión holística de las vías por las que se ha construido la relación simbiótica entre naturaleza y seres humanos (Norgaard, 1996). Una construcción que siempre ha tenido una radical historicidad y que se ha construido desde una aproximación poliédrica y diversa en los últimos decenios desde una perspectiva siempre atenta a las condiciones de reproducción material de las sociedades contemporáneas tras la revolución industrial (Sieferle, 200I).

En esta primera aproximación epistemológica, existen trabajos seminales en la década de los ochenta del siglo $X X$ que permiten atisbar rasgos clave en la agenda de trabajo de la historia ambiental, estructurada desde una clave eurocéntrica y que pueden ser consideradas como legitimantes de la modernidad capitalista. W. Cronon (1993) hace coincidir el nacimiento de esta disciplina histórica con los aires renovadores de cambios políticos y de formas de pensamiento al socaire de la crisis de la modernidad, en el contexto de los años 60. Más allá de esta contextualización, hacer historia ambiental implica considerar el peso específico de la dimensión sistémica del mundo natural (sin caer en la autorreferencia, que camina hacia una nueva parcelación del conocimiento) apostando por una síntesis en la que integrar en diálogo lo social, lo cultural y lo natural. 
Esta "impureza" metodológica facilita incorporar escalas espaciales, temporales, metodologías, estilos de comunicación y construcción del discurso que promueven la construcción de "puentes" interdisciplinares (Dovers, 2000), huyendo de reduccionismos y simplificaciones, pero con una postura crítica en la consideración del "norte académico" como único polo de creación del saber,y de la consideración del modelo civilizatorio capitalista como el objeto central de investigación de la historia ambiental, como ya indicó J. O’Connor (1997).

Historia Ambiental es un "común" que surgió y continúa como parasíntesis interdisciplinar de la historia de la biología, de la historia política, de los sistemas legales y de propiedad, de la historia social y cultural, de la historia moral. Cuando la Historia Ambiental atiende a los procesos de construcción de los estados nación en cuanto "manejadores" y "legisladores sobre el conjunto de recursos naturales en un territorio, implica prestar atención tanto a la revolución tecnológica que rediseña las formas de producción y de generación de residuos a escala global (Naredo, 2006) como a la dimensión de conflictos por los bienes que se ocasiona dentro y entre las comunidades humanas.

En resumen, Historia Ambiental — cuando tiene una profunda de matriz eurocéntrica - es la historia de la capitalización de la naturaleza, entendida esta como un reservorio de materia y energía susceptible de apropiación por individuos y/o sociedades en aras a mantener los niveles de producción y consumo del modelo capitalista. La Historia Ambiental completaría, para O'Connor, "otras historias" al insertar los conflictos socioambientales en la esfera política institucional, de las resistencias de los débiles, de las formas contrahegemónicas de lucha por la defensa del territorio. Incluso la historia cultural se redefine al apostar por un cambio de paradigma científico, pensando la representación de la tierra y del espacio desde la sustentabilidad local y apostando por el reconocimiento del "otro", del “diverso", como depositario de un saber(es) específico(s) en muchos casos ignorado (Worster, 1993, 1998).

El "mito fundacional” del pensamiento científico-ambiental de matriz occidentalocéntrica fue identificado por Rama Guha (Guha, 2000, 2006) en el trabajo de George Perkins Marsh Man on Nature: or Physical Geography as Modified by Human Action (1864). Se inicia con este trabajo una corriente científica que explora la conexión entre deforestación, desecación-sequía, y aclareo de bosques maduros para la colonización agrícola y el desarrollo industrial. En la segunda mitad del siglo XX, Rama Guha (coincidente con los trabajos antes citados de Donald Worster) indica que se ha entrado en la era de la "inocencia ecológica" y de la sociedad capitalista de consumo de masas ("affluent society") que define la satisfacción del consumo exosomático de los individuos y de las colectividades como la justificación para la posesión y uso masivo de bienes que ha puesto en el escenario del debate la actual crisis ambiental y civilizatoria en el que la historia ambiental juega un papel central. Entroncando con todo este trabajo, la historia ambiental con una matriz decolonial pretende no solo describir y cuantificar el proceso de apropiación colonial, a lo largo de siglos, de los territorios del sur, sino apostar por una visión holística del proceso de economía de saqueo-despojo (siguiendo a Harvey, 2010, 2012) que ha protagonizado el modelo civilizatorio capitalista, transformando las relaciones metabólicas de sociedades y naturaleza a escala glocal.

Desde el campo de la Historia Ambiental el concepto de extractivismo/despojo territorial es definido como transferencia de bienes naturales de manejo comunitario hacia la esfera del 
mercado, enajenando a los pueblos y beneficiando a sectores empresariales con este proceso de privatización de capital natural. De igual manera, el concepto de apropiación asimétrica implica la identificación de cómo la transferencia verificada mediante el extractivismo supone un despojo territorial que impacta sobre la arquitectura del modelo de desarrollo económico, generando episodios y realidades de desequilibrios territoriales que es práctica común de colonización del territorio que llega hasta inicios del siglo XXI como demuestra el estudio del impacto del capitalismo financiero en Baja California.

\section{Localización territorial de la investigación}

Baja California Sur tiene un área de $73.677 \mathrm{~km} 2$ que representa el 3,7\% del área total de México. Es el estado con la costa más larga, con $2.230 \mathrm{~km}$ ( $22 \%$ del total nacional), incluidas tres islas en el Océano Pacífico y más de cien islas e islotes en el golfo de California (GdC). Dispone de 690 km de longitud, $43 \mathrm{~km}$ en su parte más estrecha y $227 \mathrm{~km}$ en su parte más ancha. El golfo de California es el único mar territorial de una sola nación en el mundo asumiendo el $49 \%$ del litoral mexicano y el $50 \%$ del territorio insular nacional. Es uno de los cinco ecosistemas marinos con mayor productividad y biodiversidad del planeta y contiene, con respecto al total de especies del mundo, $40 \%$ de mamíferos marinos, $33 \%$ de cetáceos. 4.500 de invertebrados marinos, I8I de aves, 695 de plantas. En el golfo se verifica el $70 \%$ de la producción pesquera nacional y es el único lugar del mundo con cascadas submarinas.

\section{Baja California Península}

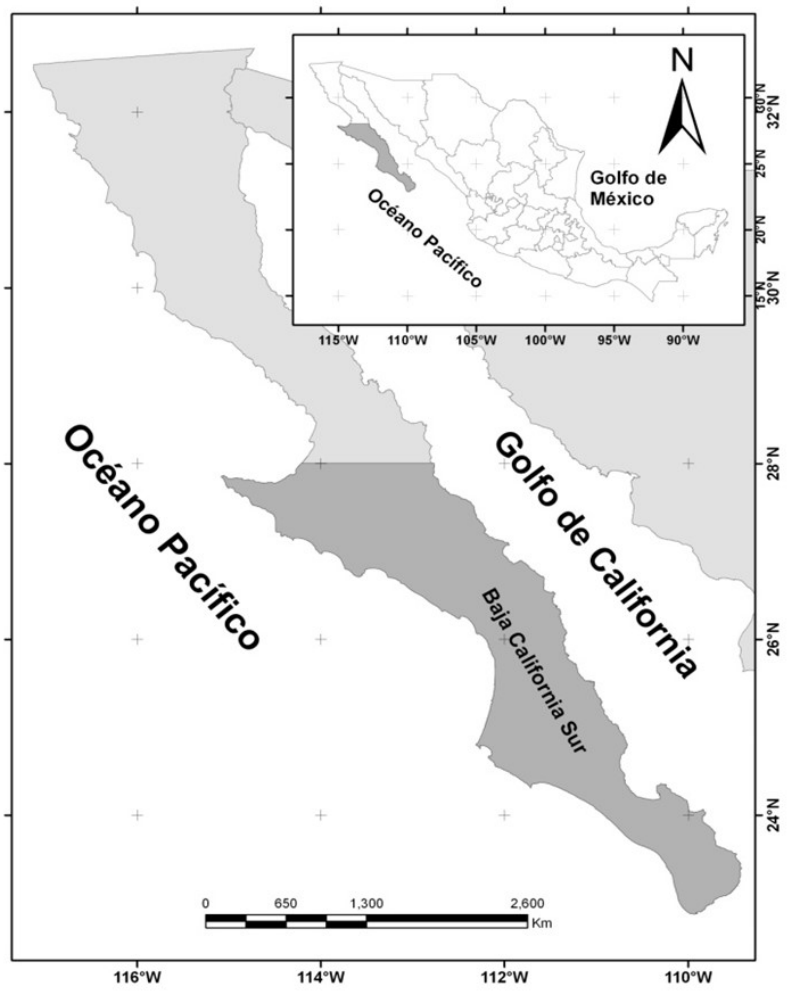

Fuente: internet 


\section{Fases de la asimetría territorial en el golfo de California. Extractivismos, saqueos y comodificación de la interacción tierra-mar}

Atendiendo al estudio-laboratorio propuesto en este artículo como sujeto de análisis, conceptualizamos al golfo de California como un espacio excepcional marcado por cuatro momentos de apropiación asimétrica que tienen lugar entre el siglo XVI y el presente, marcados por lógicas y prácticas de apropiación-despojo colonial, como evidencia la Tabla I.

En este artículo, dada la complejidad y larga duración del proceso histórico sometido a estudio, vamos a investigar de forma más profunda — no exento de continuidades a lo largo de varios de los momentos propuestos - la primera y última fase de este contínuum histórico, pero no por ello quiero dejar de consignar la importancia de los períodos centrales.

Tras el proceso de apropiación colonial, que describiré en el siguiente capítulo, en el segundo momento de apertura geográfica (entre 1830 y 2015 ) la región del golfo de California se abre entonces a la exploración de naturalistas y científicos, sobre todo estadounidenses, así como a su descubrimiento por los primeros viajeros que leen en las narraciones de esas expediciones acerca de su extraordinaria belleza y riqueza natural, de las que pueden gozar en plena exclusividad (Ortega Santos et al 2020). En el primer periodo (I830 a 1947) se llevan a cabo exploraciones naturalistas en busca de materias primas y de nuevos hallazgos para la ciencia. En este periodo se esbozan, mapean y describen las primeras rutas náuticas y terrestres para acceder a la región, se registran los puertos y sitios de anclaje seguro para la navegación recreativa, se divulgan las mejores playas y se fotografían los paisajes. Podemos decir que se forma así la antesala del turismo como práctica extractivista de territorio que se extiende desde 1960 (Ortega Santos y Olivieri, 2020). Toda esa información se usará como materia prima para el desarrollo del turismo y del mercado inmobiliario, en las aperturas siguientes. En un segundo periodo (1948 a 1972) se establecen los primeros pequeños y exclusivos resorts de playa y de pesca deportiva en la región, alimentados por un incipiente tráfico aéreo privado que se desarrolló entre Estados Unidos y México después de la Segunda Guerra Mundial. Queda así dibujado el estilo y las zonas naturales que se abrirán en las siguientes décadas a las grandes inversiones turístico-inmobiliarias. Coincide este momento con el despertar generalizado de la conciencia ecológica —en los años sesenta- que atrae la atención de más científicos y de ambientalistas, que urgen a promover la creación de las primeras áreas naturales protegidas (ANP) y los inventarios de especies, así como su protección.

En el marco de la segunda apertura geográfica se ubica un período que abarca de 1972 a 1999, clave para el devenir de las tensiones territoriales entre turismo y conservación natural, coincidente con la consolidación de la política ambiental internacional y con la construcción de la moderna infraestructura viaria y comercial que integrará el remoto territorio del noroeste - especialmente la península - en la economía nacional y del suroeste de Estados Unidos. En el contexto de la conservación auspiciada desde Naciones Unidas, el turismo aparece como una actividad económica compatible con la conservación de la naturaleza, ambos acordes al discurso del desarrollo sustentable. El golfo de California se abre entonces como un espacio turístico de la geografía mundial, incentivado además por la creación de un nuevo marco legal que facilitó desde 1980, pero fundamentalmente a partir de 1992, la liberalización de la economía nacional y con ello la apertura a los grandes capitales del ramo turístico e inmobiliario. 
Todavía en el marco del tercer momento de apertura geográfica, a partir del año 2000, se inicia la consolidación de un mercado de segundas residencias o residencias turísticas, cuyo desarrollo fue posible por el espectacular crecimiento del mercado hipotecario en Estados Unidos y la desregularización financiera que tuvo lugar el mismo año. Con ello se fortaleció la idea del golfo de California como la gran reserva natural del noroeste mexicano y destino turístico-náutico "de clase mundial". Se produce así un nuevo espacio asociado a un nuevo proyecto de poblamiento, basado ya no solamente en la venta de experiencias, sino en la venta de un estilo de vida, reordenando el territorio en función de las inversiones transnacionales y el desarrollo de numerosos proyectos de urbanización turística en los litorales. Con la llegada de los megaproyectos turísticos y turístico-inmobiliarios la alianza entre el sector conservación y el empresarial, cada uno apoyado por diferentes agencias gubernamentales, se fragiliza y a menudo estalla en conflictos. Las organizaciones no gubernamentales (ONG) y las organizaciones de la sociedad civil (OSC) que trabajan en la conservación adquieren un protagonismo cada vez mayor, incluso su poder logra mitigar la megalomanía de los planes gubernamentales y disuade el empeño de desarrolladores inmobiliarios.

Frente a este proceso de desregulación financiera territorial, que impulsa el territorio de Baja California hacia un ciclo de despojo-saqueo (consignemos también el inicio de nuevos ciclos de minería a cielo abierto como el caso de los Cardones S.A.) suponen el impulso de nuevas formas de resistencia socioambiental, de defensa del territorio, de formas de sustentabilidad que fracturan la apropiación asimétrica e impulsan formas otras de vivir en paz con el territorio, relocalizando las prácticas de producción y consumo con un menor impacto de los procesos extractivistas. 
Tabla I. Matriz Histórica de Apropiación y Comodificacion de Asimetrías. Golfo y península de Baja California.

\begin{tabular}{|c|c|c|c|c|}
\hline $\begin{array}{l}\text { Momentos de } \\
\text { investigación }\end{array}$ & Periodo & Fechas & Percepción & Apropiación \\
\hline Momento I & Colonia- mar de Perlas & $\begin{array}{l}1539- \\
1829\end{array}$ & $\begin{array}{l}\text { Descubrimiento } \\
\text {-origen golfo de } \\
\text { California. }\end{array}$ & Poder colonial \\
\hline Momento 2 & $\begin{array}{l}\text { Extractivismo: } \\
\text {-Nácar I830-I939 } \\
\text {-Sal I836-I940 } \\
\text {-Guano I856-1934 } \\
\text {-Yeso I870-20I7 } \\
\text {-Tiburón (I940-20II) } \\
\text {-Tortuga (I960-|970) } \\
\text {-Pesca industrial y } \\
\text { artesanal (I952-20I7) } \\
\text {-Acuicultura (I903- } \\
\text { I9|4 / I990-20I7) } \\
\text {-Modificación y } \\
\text { contaminación del } \\
\text { agua de mar (I938- } \\
\text { 20I7) } \\
\text {-Exploración de } \\
\text { minería submarina } \\
\text { (20I5-20I7) }\end{array}$ & $\begin{array}{l}1830- \\
2015\end{array}$ & $\begin{array}{l}\text { Stock, botín y cloca. } \\
\text { Extracción industrial } \\
\text { y comercial de } \\
\text { recursos terrestres y } \\
\text { marinos. }\end{array}$ & $\begin{array}{l}\text { Poder } \\
\text { comercial } \\
\text { de sectores } \\
\text { industriales } \\
\text { y mineros en } \\
\text { conexión con el } \\
\text { Estado. }\end{array}$ \\
\hline Momento 3 & $\begin{array}{l}\text { Período excepcional } \\
\text { con saqueo territorial } \\
\text { por actividad turística } \\
\text { y conservación } \\
\text { neoliberal que junto } \\
\text { a minería generan } \\
\text { impactos sobre } \\
\text { sustentabilidad local } \\
\text { marina }\end{array}$ & $\begin{array}{l}1960 \\
-2015\end{array}$ & $\begin{array}{l}\text { Espacio de excepción } \\
\text { y comodificación: } \\
\text { conservación } \\
\text { y turismo. }\end{array}$ & \begin{tabular}{|l|} 
Intereses \\
financieros \\
especulativos \\
en conexión \\
con gobiernos \\
federales y \\
estatales
\end{tabular} \\
\hline Momento 4 & $\begin{array}{l}\text { Rompiendo la } \\
\text { asimetría hacia la } \\
\text { sustentabilidad desde } \\
\text { las re-existencias de } \\
\text { matriz decolonial. }\end{array}$ & $\begin{array}{l}2000- \\
2015\end{array}$ & \begin{tabular}{|l|} 
Resignificación \\
territorial e identidad: \\
actores y procesos \\
biorregionales.
\end{tabular} & $\begin{array}{l}\text { Acciones } \\
\text { colectivas y } \\
\text { locales de } \\
\text { desarrollo } \\
\text { comunitario. }\end{array}$ \\
\hline
\end{tabular}




\section{Extractivismos océano-territorial. Colonialidad de la naturaleza en el mundo moderno y contemporáneo.}

\section{I. Primer momento de apropiación asimétrica en el golfo de California. Mar de Perlas}

El primer contacto entre californios y españoles sucedió en I533, en el contexto de las expediciones que se realizaron por orden de Hernán Cortés a la mar del Sur,"porque había fama de ricas islas de perlas" (Díaz del Castillo, I568, p. 980). Fortún Ximenez, al llegar a las costas peninsulares vio que los indígenas llevaban perlas trenzadas en sus cabellos y de inmediato intentó hacerse con ellas, lo que provocó agresiones y finalmente la muerte de algunos tripulantes de la amotinada flota. Los sobrevivientes regresaron a la Nueva España y la noticia del descubrimiento de la "isla de las perlas" rápidamente se expandió. Ante esta perspectiva, y harto de la ineficiencia de sus marinos, Cortés decidió encabezar personalmente la cuarta de las expediciones que emprendió para conquistar las riquezas que se creía que había en la mar del Sur (Del Río, 1990; Mathes, 1973). Así, a partir de 1586, la Corona, en tanto que propietaria de los placeres perleros, estableció mediante una licencia real la autorización de pescar perlas en el marco de la legalidad, pero con una doble condicionante, el pago del "quinto" de perlas y el compromiso de los detentores de la licencia de realizar la demarcación, la exploración y la protección de las costas del Golfo y del Pacífico.

La licencia o "asiento" para la pesca de perlas —único medio legal para realizar esta actividad - otorgaba a su detentor la exclusividad de la explotación de los placeres perleros por un tiempo limitado y con ciertas obligaciones. Si los informes sobre las costas de California no eran satisfactorios, o la cantidad devengada por el quinto de perlas no era considerada suficiente, cuando se cumplían los pocos años de prueba indicados en el contrato que acompañaba a la licencia, esta era retirada de su poseedor y entregada a un nuevo solicitante. La organización de la pesca de perlas fundamentada en el trabajo de las armadas prácticamente no sufrió variaciones desde el siglo XVI hasta el XX, cuando se agotaron los placeres. No obstante, el más importante de estos fue el causado por la extinción de los buzos californios, ya a finales del siglo XVIII; pero en el periodo que ahora nos ocupa, uno de los componentes más interesantes del trabajo de las armadas fue precisamente el de las negociaciones que se establecieron para la pesca de perlas entre esos nativos y los armadores. La síntesis que enseguida presentamos respecto a la organización de la pesca de perlas proviene fundamentalmente de tres fuentes: la crónica de Miguel del Barco de 1780, las relaciones de Isidoro Atondo y Antillón (Atondo y Antillón), 1685; y, entre otros estudios, un artículo del historiador Peter Gerhard (1956).

La armada se dirigía a ciertos sitios en las costas occidentales del Golfo, donde sabían que encontrarían californios interesados en hacer el trueque o la pesca. Primero se intentaba cambiar ciertos objetos codiciados por los nativos peninsulares por las perlas que ellos tuvieran en su posesión. Una vez fijado un trueque entre colonizadores y pueblos originarios (guaycures, pericúes y cochimíes), comenzaban las negociaciones para llegar a un acuerdo en el que los buzos extraerían conchas para el armador, a veces dificultado por la comunicación lingüística. Los armadores debían retribuir a los californios por su trabajo ya sea en conchas cerradas o en objetos a cambio de perlas. Cuando se acordaba el primer tipo de remuneración, el armador 
recibía del buzo tres de cada cinco ostras extraídas, dos para la armada y una para el rey. Después, se procedía a la apertura de las conchas, el armador guardaba las perlas contenidas en sus conchas y ponía en un cofre especial las que hubieran contenido las conchas seleccionadas para el rey, después procedía a negociar el trueque de las perlas que hubieran contenido las ostras que había recibido el buzo. Cuando se elegía el segundo tipo de acuerdo, antes de la pesca se determinaba un lote de ciertos objetos a cambio de cierta cantidad de ostras extraídas. En este caso, el armador se quedaba con todas las ostras y sus perlas, a excepción de la quinta parte para el rey. A partir de 1586, la legislación para la pesca de perlas, junto con el establecimiento de la licencia, prohibió el maltrato a los indígenas, tanto a los buzos como a los demás miembros de sus grupos. Sin embargo, todas las crónicas reportan la frecuencia y crueldad con la que sucedían los maltratos. También es posible percibir que estos aumentaban a medida que las perlas escaseaban, como resultado de la desesperación de los armadores que no lograban satisfacer sus ambiciones.

La primera licencia para la pesca de perlas fue la concedida por Felipe II a Hernando de Santotis, Antonio de Castillo y Pedro Lobato del Canto el 15 de junio de 1586. El documento establecía que el virrey podía "otorgarles licencia para que, en las costas del mar del Sur, desde el puerto de Natividad hasta California, puedan negociar, comerciar y pescar en lo que ya está descubierto, durante diez años, pagando la vigésima parte de todo lo que extraigan y dentro de tres años ellos tendrán mi confirmación respecto a dicha licencia” (Vásquez, 1586, p. 14).

En 1592 Sebastián Vizcaíno (llegado a Nueva España como soldado de fortuna en I583) recibió la segunda licencia para la pesca de perlas y formó una compañía con once asociados para financiar su vasta empresa. Vizcaíno solicitaba el derecho exclusivo por veinte años para la pesca de perlas, de atún, sardina, bacalao y otros pescados, así como para explotar los yacimientos de sal, oro y plata en la región del puerto de Natividad a la California. Para llevar a cabo esta jornada, se comprometía a llevar "rastros para la pesquería de perlas, chinchorros para el pescado, mineros para descubrir minas y aderezo para fundir metales", asimismo, asumiría el compromiso de "descubrir toda la ensenada y boca de las Californias y de toda ella tomar posesión por vuestra majestad, poniendo puertos de mar, cabeceras y ciudades en la real corona". Siguiendo la legislación vigente, todo esto lo ejecutaría Vizcaíno "lo más quietamente y sin hacer agravio a los naturales [...] con buenos modos y dádivas para traerlos al servicio de Dios y vuestra majestad" (Mathes, 1973, p. 32).

Las peticiones de Vizcaíno fueron concedidas, se le otorgó la licencia para la pesca de perlas y la extracción de metales preciosos de lo que debía pagar la quinta parte, de los pescados salados y empaquetados solo debía pagar la décima. Esta compañía debía probar su capacidad en los primeros cuatro años de operaciones a partir de marzo I594. Si lograba instalar una colonia en ese plazo, el contrato adquiría validez por los siguientes diecisiete años, concediéndole la exclusividad de la extracción de perlas y pescado en la zona asignada (Campos Guerrero, I593, Pp. 96-I I6). Los términos en los que se estableció esta licencia muestran claramente el concepto de riesgos compartidos entre una empresa privada y el estado colonial. El 3 de septiembre de 1596, Vizcaíno arribó a los parajes donde Cortés había realizado sus primeros reconocimientos, región a la que denominó Nueva Andalucía, estableciendo el Puerto de La Paz. Sin embargo, un mes después, los colonos - temiendo por sus vidas - le suplicaron regresar debido a la aridez del entorno y a la agresividad de los hambrientos indígenas. El capitán hizo caso omiso y continuó la 
extracción perlera que había funcionado correctamente. No obstante, un incidente con un buzo fue motivo suficiente para que las hostilidades estallaran. Las armadas tuvieron que retornar a La $\mathrm{Paz}$, se montaron empalizadas y las agresiones se saldaron con un gran incendio en el campamento, lo que forzó a los expedicionarios a volver a Nueva España a finales del año 1596.

El I 3 de agosto de $16 \mathrm{I} \mathrm{I}$, una compañía formada porTomás de Cardona y Asociados obtuvo la autorización real para proceder a la pesca de perlas en California, Panamá y las Bermudas. En la Nueva España esta compañía estaba a cargo de Nicolás de Cardona (sobrino del citado Tomás), Juan de Iturbide y Pedro Álvarez Rosales. Los preparativos para la expedición llevaron varios años, pero cuando por fin estuvo todo listo para realizar el primer viaje, las expectativas fueron frustradas a causa del ataque del pirata holandés Joris van Spilbergen, que capturó el navío San Francisco cuando regresaba cargado de perlas. Las grandes pérdidas desalentaron a los Cardona para continuar con la pesca en el golfo de California y prefirieron continuar en el de Panamá. Se inició un período de reclamación sobre los derechos de exclusividad para la pesca de perlas que continuaron hasta I63 I cuando la licencia de perlas finalmente fue concedida a Francisco de Ortega (León-Portilla, 2000).

Ortega era un constructor de navíos, por lo que aseguró al virrey marqués de Cerralvo que podía realizar cuantos viajes fueran necesarios a California para conocer en detalle sus costas e identificar el mejor emplazamiento para establecer una población y practicar la pesca de perlas. En los años siguientes, Ortega y su piloto Esteban Carbonel realizaron tres viajes a California, a pesar de la prohibición temporal establecida en 1634 a causa de nuevos litigios en torno a los derechos sobre la licencia para la pesca de perlas. Los viajes de Ortega finalizaron cuando en 1636 su barco naufragó en uno de los bancos de arena de la bahía de La Paz, aunque avanzó información utilizada en posteriores expediciones auspiciadas por la Corona (León-Portilla, 2000, pp. I5 I- I88).

Será necesario llegar al siglo XIX, cuando la extracción de nácar y perlas y el comercio de ambos productos era la actividad económica más importante del golfo de California. En La Paz y Mazatlán se concentraban toneladas de conchas, limpias y empacadas para ser exportadas a los EE.UU. y a Europa (Esteva, 1977, p. 33), donde se apreciaba mucho la calidad del nácar de madre perla del golfo de California. Las perlas se vendían casi en su totalidad en París, que era entonces el centro del mercado mundial de estas codiciadas gemas. El contrabando de estas fue la norma ya que debido a su pequeño volumen era fácil disimularlas, no sucedía lo mismo con los pesados y voluminosos sacos de conchas; aunque también hubo forma de comercializarlos sin pagar los impuestos requeridos por las aduanas marítimas. De cualquier forma, legal o ilegalmente, la comercialización de ambos productos fue la base del enriquecimiento de buena parte de las familias de la élite regional. Sin embargo, la intensificación de la pesca, entre 1874 y 1884 , a causa del incremento del número y tamaño de las armadas, y mediante el buceo de chapuz atrajo nuevos empresarios perleros, tanto nacionales como extranjeros. Entre 1876 y 1883 se solicitaron más de 70 licencias anuales para la explotación de los placeres perleros (AGN, I877; AHPLM, I879, 1884).

Las concesiones territoriales para la colonización fueron un mecanismo ideado para superar dos carencias constantes de la joven nación mexicana: la escasa población y la falta de capitales. El 30 de marzo de 1864, Benito Juárez firmó un contrato con la compañía colonizadora de Jacobo 
P. Leese, concediéndole los terrenos ociosos desde el paralelo $24^{\circ} 20^{\prime} \mathrm{N}$ hasta el $31^{\circ} \mathrm{N}$ (Valadés, 1974, p. 187) obligándola a pagar solo cien mil pesos, pero comprometiéndola a establecer un mínimo de doscientas familias. La concesión no cumplió con sus compromisos, pero si aprovechó una de las cláusulas de su contrato que le permitía la explotación de los recursos naturales que se encontraban en su territorio para explotar la orchilla, de lo cual sacó grandes beneficios (Cariño, 1996, pp. 39-42).A esta compañía siguieron otras semejantes, en su mayoría en manos de extranjeros, lo que provocó amplio malestar entre los sudcalifornianos. No obstante, la existencia de esta política sugirió a los grandes empresarios perleros una estrategia que podía beneficiarles.

En sintonía con esta colonización de la naturaleza, auspiciada por el estado nación tras la independencia, entre 1884 y 1906 el Ministerio de Fomento, en representación del ejecutivo federal otorgó 26 concesiones territoriales en el golfo de California para la extracción de nácar y perlas. Los primeros beneficiarios de esta política fueron los empresarios locales, quienes recibieron las primeras seis concesiones todas ellas ubicadas en la costa sudcaliforniana donde se localizaban los mejores placeres desde San José hasta el norte de la Bahía de Loreto y en las islas aledañas.

Las concesiones eran hechas a personas o a compañías, el territorio asignado en concesión no se traslapaba entre los contratos, por lo que esta política puede ser considerada como una forma de ordenamiento del territorio marino, lo que permitía al gobierno federal tener mayor control sobre los recursos perleros. Siempre debía haber un signatario que representara a la empresa y que se responsabilizara del cumplimiento de las cláusulas de los contratos (Cariño, 1998 , pp. 694-704). El poder sobre las actividades de extracción de las ostras perleras que ostentaba esta compañía creció en proporción al incremento del territorio marino bajo su concesión y del capital invertido en la empresa. Esto sucedió en las dos etapas de fusión/ampliación de la concesión, primero en 1893 cuando se formó la Compañía Perlífera de la Baja California Sucesores y en 1897 cuando esta vendió su concesión a The Mangara Exploration Limited. El capital de la primera era también estadounidense, como el de su predecesora, y el contrato fue firmado en San Francisco; su representante legal en La Paz era Miguel L. Cornejo. El capital de la segunda era británico, el contrato de transferencia de la concesión territorial y de venta de todos los bienes de la compañía, fue firmado en la Ciudad de México; sus representantes legales fueron entonces los hermanos Miguel L. e Ignacio Cornejo (AHPLM, I897).

La Mangara fue fundada en Londres el 30 de junio de 1897 con un capital equivalente a ciento cincuenta mil pesos. Con esta concesión el gobierno federal puso en manos de los ingleses el control de todos los recursos perleros mexicanos y de todos los litorales del Pacífico, desde la frontera con los EE.UU. hasta la barra del Suchiate. En 1899 La Mangara amplió su territorio concesionado anexando las islas Marías. En 190I, bajo la representación de Joaquín D. Casasús (prominente intelectual y funcionario, allegado a Porfirio Díaz), logró la prorrogación de su contrato por siete años más.Al igual que las otras compañías perleras, La Mangara tenía una serie de obligaciones en las que destacaba el cuidado y reproducción de los placeres perleros, pero esta empresa nunca cumplió sus obligaciones. Por tales motivos el gobierno hubiera podido rescindir su concesión, pero su gran poder económico y político le otorgó una completa impunidad. Por el contrario, en 1908, y también representada por Casasús, logró nuevamente la renovación de su concesión, con la prorrogación de su contrato hasta 1916 (AGN, 1905). 
En 191I, La Mangara cedió autorizando los contratos de subarrendamiento en su territorio; pero las condiciones de estos eran aún más leoninas que las de compañías precedentes. Solo duraban dos meses, los armadores debían pagar 125\$ pesos mensuales por cada escafandra empelada, debían vender a La Mangara todas las conchas extraídas a $5 \$$ el quintal las de primera categoría y a solo dos centavos las de segunda. También debían entregar a la compañía el $10 \%$ del nácar y de las perlas obtenidas. La Mangara se reservaba además el derecho de pescar en las zonas subarrendadas. Su única obligación consistía en pagar a la aduana marítima el impuesto sobre las conchas extraídas. Poco tiempo después, el derecho por cada escafandra empleada ascendió a $500 \$$ pesos y el precio fijado para comprar la concha extraída quedaba a consideración exclusiva de la compañía.

En vez de aliviar la tensión social, estas medidas la agravaron,y en junio de I9I I desembocó en una serie de acciones tendientes a resistir a las imposiciones de La Mangara. Los trabajadores de la compañía organizaron una huelga para protestar contra los bajos salarios, incitaron a la población a desconocer la exclusividad territorial de la compañía ejerciendo libremente la pesca y se manifestaron frente a las oficinas del representante local de La Mangara, FrankW. Moore, que era también vicecónsul de Gran Bretaña. El movimiento por la liberación de la pesca debe ser considerado como la primera manifestación revolucionaria del distrito sur de la Baja California y como tal no tuvo solución bajo el porfiriato. Venustiano Carranza en julio de 1916 estableció el primer reglamento posrevolucionario para la extracción de ostras perlas. La licencia costaba $100 \$$ pesos y debía ser tramitada por un abogado en la Ciudad de México ante el Ministerio de Fomento. Posteriormente, por cada tonelada de concha debía pagarse un impuesto de $40 \$$ pesos y el $10 \%$ de las perlas que se hubieran encontrado.

Para entender el decaimiento de la explotación de ostras perleras, es necesario considerar la evolución del mercado de nácar y perlas entre 1915 y 1940. Las dos guerras mundiales y la crisis financiera de finales de los treinta y principios de los cuarenta provocaron la caída de la demanda de los productos suntuarios. Sin embargo, también en esa década las perlas de cultivo se abrieron paso en el mercado mundial, primero tímida y discretamente, pero a partir de 1940 de forma abierta y masiva. Por el contrario, la demanda de nácar que inició su descenso durante la Primera Guerra Mundial nunca se recuperó.Al salir de la guerra, la industria que empleaba el nácar como materia prima lo substituyó con materiales artificiales y este quedó relegado al limitado papel de ornamento decorativo que había tendido antes del siglo XIX.

El impacto de estas variaciones del mercado mundial sobre la extracción de los placeres perleros del golfo de California fue un retroceso a la situación anterior a 1830 y paulatinamente las grandes armadas perleras fueron abandonando sus actividades a partir de 1930.

\subsection{Capitalismo Financiero y Despojo Territorial en Baja California Sur}

En el marco del proceso de apropiación asimétrica del territorio sudcaliforniano que describía en la tabla I, desde mediados del siglo $X X$ se implementa una nueva dinámica económica de saqueo, protagonizado por el aumento del turismo como palanca de desarrollo económico. Este sector económico que protagonizó hegemónicamente los discursos oficiales e internacionales en las políticas económicas, no se impregnó de prácticas ni estrategias vinculadas a la protección 
de la naturaleza, en sus diferentes acepciones, que también estaba articulada en los discursos y legislaciones tanto del Estado Mexicano como de Naciones Unidas.

Con la creación a partir de 1974 del FONATUR (Fondo Nacional de Fomento al Turismo, fideicomiso del gobierno federal administrado por la Secretaría de Hacienda y Crédito Público y Nacional Financiera', y la Ley Federal de Fomento al Turismo junto a la creación de la Secretaría de Estado (Baños Francia, 2012, p. 43), se pusieron en marcha instituciones con facultades para expropiar tierras para potenciar el desarrollo turístico en nombre del interés público y para establecer reservas territoriales con la finalidad de ofrecerlas a inversionistas como suelo urbanizable en Loreto y Los Cabos, BCS, y en Bahía Banderas, Nayarit, abriendo la posibilidad de crear un multimillonario negocio con la venta del suelo. Los dos primeros megaproyectos turísticos-inmobiliarios en la región del GdC fueron Loreto y de Los Cabos, planeados como lujosos destinos internacionales de golf y pesca deportiva. En Loreto, donde FONATUR adquirió grandes reservas territoriales, el modelo de desarrollo turístico en formato de corredor costero (Proyecto NOPOLO) no prosperó.

Las medidas de expropiación de FONATUR se intensificaron en los años ochenta. En 1987, mediante decreto presidencial, se autorizó a FONATUR a "realizar todas las operaciones de enajenación, administración y concesión de los derechos de aprovechamiento de las superficies susceptibles de explotación económica directa, conforme se vayan realizando las obras" (DOF, 1987). Comenzó una nueva proyección de Baja California Sur (BCS) como destino turísticonáutico, con fe en que sería el sector que lograría integrar finalmente a la región en un plan nacional de desarrollo que estimularía el ansiado crecimiento económico y el poblamiento del territorio. Con la reforma al artículo 27 constitucional en 1992, para liberar la propiedad ejidal, inició una transformación de la base agraria del país y los ambicionados terrenos costeros comienzan a ofrecerse como tierras de inversión en el mercado inmobiliario internacional. Se crean así los nuevos espacios turísticos en las costas del GdC y la propiedad con frente de playa adquiere un lugar protagónico en la economía regional. En 200 I, el gobierno federal, los gobiernos de Baja California, Baja California Sur, Sonora y Sinaloa, conjuntamente con distintos organismos empresariales, celebraron un convenio para poner en marcha el tan anhelado proyecto ENMC (Escala Náutica Mar de Cortés), utilizando el "Mar de Cortés" como marca integradora e ícono del turismo náutico en el país. Un megaproyecto cuya finalidad consistía en multiplicar las oportunidades de inversión.

Se previó que la ENMC llegaría a consolidarse en 2015, complementándose en veinticinco años mediante el desarrollo de tres programas: I) el desarrollo de las 28 escalas náuticas, 2) el mejoramiento integral de 21 localidades rurales costeras, y 3) la planeación de rutas y circuitos turísticos, acompañados de un programa de promoción nacional e internacional. El Programa de Mejoramiento Integral de las Localidades Costeras tenía como objetivo reordenar y poner en valor los poblados mediante la ejecución de "Proyectos Integrales" o "Desarrollos Turísticos Integrales" (DTI).

Esta urbanización turística debe ser comprendida como parte de un proceso global de

Con el fin de facilitar el crecimiento del turismo, el Banco Central en colaboración con Nacional Financiera (NANFIN) había creado el Fondo de Infraestructura y el Fondo para la Garantía y Promoción del Turismo que se fusionaron para crear el FONATUR. 
desregularización del sector financiero, con un efecto en el inflamiento del sector inmobiliario. La apertura de la región al capital financiero e inmobiliario, y con ello a la conversión de los enclaves turísticos hacia los enclaves residenciales, fue posible por dos sucesos importantes que marcaron el destino de las finanzas mundiales: la revocación de la Ley Glass Steagall y el recorte de las tasas de interés por la Reserva Federal de Estados Unidos. Por otro lado, la reducción de las tasas de interés, motivada por la burbuja tecnológica de 1999-2000, vinculada con el crecimiento de las empresas relacionadas al internet, propició la expansión del crédito, especialmente del crédito hipotecario (Harvey, 2010 y 20I2). Entre 2004 y 2010 se identificó tan solo en BCS la participación de 19 intermediarias financieras no bancarias que ofrecían créditos hipotecarios transfronterizos. Con carteras de crédito que oscilaban entre los 100.000 y 5 millones de dólares (USD) y períodos de pago de 20 a 30 años, las intermediarias financieras no bancarias asumieron en el mercado de la vivienda turística de México el riesgo por el pago de 7,99\% de interés².

El enlace entre el tercer y cuarto momento de apropiación asimétrica, citados en la tabla I, se revela en los movimientos de la sociedad civil orientados a crear espacios y prácticas de resistencia socioambiental frente a los episodios de despojo territorial y la inviabilidad/ insustentabilidad del modelo económico extractivista. Surgió así la Alianza para la Sustentabilidad del Noroeste Costero Mexicano (ALCOSTA), una asociación civil conformada por 2I OSC que trabajan en distintos procesos de conservación en la región del GdC. Motivadas por la coyuntura y el financiamiento internacional que se ofreció para regular los procesos de crecimiento costero y conservación urgentes en la región del Golfo, iniciaron un proceso de consulta pública con autoridades estatales, municipales, comunidades costeras y empresariales para demostrar la inviabilidad ambiental de las escalas náuticas, incidiendo en la cancelación de la ENMC. Demostraron que el mercado turístico-náutico estaba sobreestimado en un $474 \%$, respaldándose con ello argumentos respecto a la insustentabilidad económica de la ENMC. Como resultado se dejaron de privilegiar las rutas náuticas como elemento central del megaproyecto, priorizándose consecuentemente el sector turístico-inmobiliario. De las veintiocho marinas que se propusieron diez fueron realizadas y de estas, solo ocho entraron en operación. El conjunto de organizaciones recomendó también impulsar un ordenamiento ecológico regional del GdC, a la vez que los ordenamientos locales donde se planeaba desarrollar las localidades turísticas (Aguirre Muñoz, 2002). De todos los estados que integran la región del GdC, BCS es donde se ha propuesto la construcción del mayor número de DTI o megaproyectos de urbanización turística. Mientras que entre 2003 y 2010 se planearon 45 DTI, en 2019 este número ascendió a 63 megaproyectos.Todos ellos con un frente de playa de al menos $300 \mathrm{~m}$, que se suma a los $347 \mathrm{~km}$ antes mencionados. Inevitablemente, tratándose de propiedad privada frente al mar y tomándose en consideración que no hay regulaciones en México que protejan el espacio costero y las playas más allá de los 20 $\mathrm{m}$ que conforman la ZOFEMAT, este modelo de crecimiento está provocando la asfixia costera y el cerramiento generalizado del acceso de la sociedad al mar. Para analizar este proceso algunos autores utilizan el término coastal grabbing (Bavinck et al., 2017) otros el de ocean grabbing (Foley y Mather, 2019; Bennett, Govan y Satterfield, 2015), que puede traducirse como acaparamiento marino-costero.

Esto se refiere en términos generales al cerramiento del mar y a la apropiación por

\footnotetext{
2 Información obtenida de la consulta de los sitios de las propias intermediarias financieras no bancarias, las agencias de bienes raíces y
} consultoras de mercado de la vivienda turística en México. 
agentes externos del espacio y de los recursos marino-costeros, que para lograr objetivos de conservación y de manejo e implementar planes de desarrollo, tienden a prácticas de control y dominio que conllevan al despojo y al desplazamiento de las comunidades costeras y usuarios locales.

En 2006 el Grupo Grand Coral Los Cabos propuso desarrollar un megaproyecto turísticoresidencial con una oferta hotelera y residencial de 36.500 apartamentos y un campo de golf. De acuerdo con investigaciones realizadas por la prensa española en 2012, los inversionistas y promotores principales eran directivos de sociedades financieras bancarias en España. Entre ellas la financiera Bancaja Hábitat, que creó en 2003 una sociedad de promoción turística llamada Valfensal ${ }^{3}$ para extraer capitales del banco Caja de Ahorros del Mediterráneo (CAM) que se asoció también al megaproyecto junto con un grupo de empresarios españoles. Para vehicular la inversión tomaron participación en la sociedad holandesa Grand Coral que a su vez controlaba la mercantil mexicana Inmobiliaria Piedras Bolas, propietaria de dos activos de interés para el grupo Grand Coral: un terreno urbanizable de 400 ha con $2 \mathrm{~km}$ de playa y otro aledaño de 870 ha (Piedras Bolas) (Altozano, 2012). De acuerdo con una auditoría realizada por el Banco de España en 2009 , se estipuló que debido a la dependencia del desarrollo de una carretera para hacer factible la inversión y obtener retornos de los créditos proporcionados por la banca, se trataba de una inversión con riesgos. Para no poner en mayor riesgo las inversiones, Bancaja se comprometió a invertir cincuenta millones de dólares (USD) para la construcción de la carretera, pero al ser negada dos veces por la SEMARNAT (Secretaría de Medio Ambiente y Recursos Naturales), en 20II y 2012, el megaproyecto Grand Coral quedó en el limbo. En 2012 los directivos de las financieras Bancaja, CAM y Valfensal fueron investigados por el Fondo de Reestructuración Ordenada Bancaria acusados por apropiación indebida, administración desleal, blanqueo de capitales y delito fiscal (El País, 20I2).

Un proceso similar al antes descrito acaeció a partir de 2007 cuando se creó la entidad especulativa financiera Hansa Baja Investments. Lo que sigue después de su puesta en marcha fue un complicado entramado de participación de entidades financieras e inmobiliarias que facilitaron grandes flujos de capitales que fueron auditados por la Banca de España tras la crisis inmobiliaria ${ }^{4}$. El resultado fue el traslado de la inversión del plan maestro Cabo Cortés (en plena Reserva de la Biosfera Cabo Pulmo), reestructurado y gestionado en 2012 como Cabo Dorado, y con ello la transferencia de la propiedad del suelo a las compañías chinas Beijing Sansong International Trade Group, asociada a la estadounidense Glorious Earth Group, y China State Construction Engineering Corporation; empresas vetadas y acusadas de fraude por el Banco Mundial. En 20I4 se volvió a solicitar permiso para la construcción del DTI y por tercera vez la SEMARNAT, contando con un fuerte apoyo técnico y legal de la sociedad civil organizada, negó el permiso argumentando los impactos socioecológicos. La movilización ciudadana que inició localmente, se extendió a nivel nacional y evidenció la insostenibilidad ambiental del megaproyecto Cabo Cortes

\footnotetext{
Administrada por Juan Vicente Ferri, director del grupo hotelero Marconfort.

La inversión fue promovida y facilitada por John McCarthy, representante de Hansa Baja Investments, quien tuvo una participación en la inversión siendo director de FONATUR, que después de la crisis inmobiliaria fue reestructurado y renombrado Cabo Dorado (Garduño, 2008). En 1997 fue presidente de Raintree Vacation Club, subsidiaria de la estadounidense Raintree Resorts International, dedicada al desarrollo de propiedades vacacionales en México. En 1997 adquirieron la División de Turismo del grupo financiero Bancomer, de la cual McCarthy fungió el mismo año como presidente y director general. De acuerdo a una investigación realizada por Greenpeace España, titulada "Cabo Cortés: destruyendo el paraíso", Hansa Baja Investments fue investigada por la autoridad española por delitos contra Hacienda Pública y falsificación de documentos (Greenpeace, 2010). En 2012 Hansa Baja Investments cambia su razón social a La Rivera Desarrollos BCS.
} 
y luego Cabo Dorado, colocando los planes de crecimiento costero de la zona en el ojo de la prensa nacional e internacional.

Tan solo en BCS, de 2004 a la fecha, se han identificado veintiún conflictos socioambientales por el desarrollo de un megaproyecto de urbanización turística ${ }^{5}$. De todos los conflictos socioambientales identificados, únicamente se resolvieron dos. Los demás son latentes o están vigentes, y dieciséis de ellos tienen como detonante el acceso y apropiación de las playas (Valiente, 2020). No obstante, para tener una idea de la magnitud de esta problemática, el Observatorio de Violencia Social y de Género de la Universidad Autónoma de Baja California Sur dio a conocer en 2010, que entre 2005 y 2008 las agencias del Ministerio Público en BCS atendieron II59 denuncias de despojo de tierras, reconocidos en la ley como delitos patrimoniales. Esta cifra puso a BCS en el segundo lugar de delitos por despojo territorial en el conjunto del país (Uribe, 20I0).

\section{Conclusión}

A modo de resumen, el texto presentado revela varias ideas fuerza. En primer lugar, el trabajo de la historia ambiental marina avanza nuevos estudios sobre el impacto de los modelos extractivistas que tanto la Corona como los estados nación imponen, atendiendo en ambos casos a un proceso de enajenación-privatización del uso de los recursos terrestres y marinos tanto para facilitar un acceso oligarquizado y desigual a los mismos, para los habitantes del territorio sudcaliforniano. En segundo lugar, este trabajo permite una consideración ajustada de lo que se denomina como acumulación por desposesión (citando a Harvey) no solo en perspectiva actual, sino enriquecido con una mirada temporal más amplia, a lo largo de varios siglos,y nacida del proceso de colonización imperial pero inserta en la matriz productiva del capitalismo como modelo civilizatorio.

En tercer lugar, desde la historia ambiental se avanza hacia una historia decolonial ambiental (ya emergente) en el que consignar las formas de resistencia histórica y actual de los grupos humanos contra una economía de saqueo, de largo recorrido temporal, estableciéndose una investigación sobre conflictos socioambientales que facilite el diálogo epistémico entre historia ambiental y ecología política, punto de intersección nacido de la mirada atenta a las situaciones histórica de injusticia socioambiental. El golfo de California, con sus potencialidades ambientales ya descritas, aparece como un laboratorio excepcional para el estudio de los procesos de colonialidad territorial desde el siglo XVI hasta el siglo XXI, comprobando la interfase tierramar en monocultivos de extracción e inputs para el modelo fabril o de consumo global. Para ello es central la aportación de este estudio, que pone atención a una historia ambiental oceánica, convertidos en espacios de flujos biológico-económicos que vertebran desigualdades Sur-Norte Global. Como corolario, la historia ambiental puede aportar nuevos semilleros de investigación para ampliar marcos referenciales de la injusticia ambiental dentro del geopolítica global.

Puede consultarse también un estudio realizado por la alianza de 2I OSC, ALCOSTA (2006) para monitorear los impactos ambientales, sociales y económicos del proyecto Mar de Cortés en 48 comunidades costeras del golfo de California. Una de las observaciones que destaca en el informe es la denuncia de la transformación de la franja costera por la actividad inmobiliaria, no por el turismo (p. 10). 


\section{Referencias}

AGN (I877). México 1877, Memoria de Hacienda y Crédito Público I876-I877. Archivo General de la Nación.

AGN (1905). Diario Oficial. México, 6 de enero de 1905, núm. I5. Archivo General de la Nación.

Aguirre Muñoz, A. (ed.) (2002). Escalera Náutica del Mar de Cortés: Reorientación hacia la Sustentabilidad. La Paz. En https://acortar.link/Nxhs9

AHPLM (I879). La Paz. Hacienda, vol. I50, Exp. 3. Archivo Histórico Pablo López Martínez, La Paz, BCS, México.

AHPLM (I884). La Paz. AJDBCS, Procesos Penales, Exp. 27. Archivo Histórico Pablo López Martínez, La Paz, BCS, México.

AHPLM (I897). México I897,AJDBCS Procesos Penales, Exp. 6/90I.Archivo Histórico Pablo López Martínez, La Paz, BCS, México.

ALCOSTA (2006) ¿Desarrollo turístico regional? Monitorio de los desarrollos turísticos e inmobiliarios costeros del Noroeste de México 2005-2006. Mazatlán:Alianza para la sustentabilidad del Noroeste Costero,A.C.

Altozano, M. (9.1I.20I2). Una sociedad opaca para saquear la CAM. El País.

Atondo y Antillón, I. [1685] (1974).Autos sobre los parajes que ha descubierto en las Islas Californias el almirante don Isidro de Atondo; y la última entrada que está para ejecutar en ellas. En Mathes, W. M. (Ed.), Californiana III. Documentos para la Historia de la transformación colonizadora de California (I 679-I 668), tomo II, (pp. 463-559). Madrid: Ediciones José Porrúa Turanzas.

Baños Francia, J.A. (2012). Ocupación del territorio litoral en ciudades turísticas de México. Revista Bitácora Urbano Territorial, 20 (I), 4I-52.

Bavinck, M., Berkes, F., Charles,A., Dias,A. C. E., Doubleday, N., Nayak, P., and Sowman, M. (2017). The impact of coastal grabbing on community conservation-a global reconnaissance. Maritime Studies, 16 (8), I- 17.

Bennet, N. J.; Govan, H., \& Satterfield,T. (20I5). Ocean grabbing. Marine Policy, 58, 6I-68.

Campos Guerrero, P. [1593] (1965). Asiento que tomó el virrey de Nueva España don Luis de Velasco con Sebastián Vizcaíno y otros armadores compañeros suyos para la jornada al descubrimiento de la California. En Mathes, W. M. (Ed.), Californiana I. Documentos para la Historia de la demarcación comercial de la California (I583-I632), tomo I, (pp. 96-I I6). Madrid: Ediciones José Porrúa Turanzas.

Cariño, M. (1998). Les mines marines du golfe de Californie, Histoire de la région de La Paz à la lumière des perles. (Tesis de Doctorado en Historia y Civilizaciones). París: École des Hautes Études en Sciences Sociales.

Cronon,W. (1993). The Uses of Environmental History. Environmental History Review, I7 (3), I-23.

Del Río, I. (1990). A la diestra mano de las Indias. Universidad Nacional Autónoma de México, Instituto de Investigaciones Históricas.

Díaz del Castillo, B. [1568] (20I I). Historia verdadera de la conquista de la Nueva España. Edición, estudio y notas de Serés, G. Madrid: Real Academia de la Historia.

DOF Diario Oficial de la Federación (1987). Decreto por el que se desincorporan del régimen de los bienes del dominio público de la Federación, las superficies ganadas al mar que se señalan y se autoriza su aportación para incrementar el patrimonio del Fideicomiso de la Ciudad El Recreo-La Paz (FIDEPAZ), Secretaria de Desarrollo Urbano y Ecología.

Dovers, S. (2000). On the contribution of Environmental History to Current Debate Policy. Environment and History, $6(2),|3|-|5|$.

Esteva, J. M. (1977). Memoria sobre la pesca de la perla en Baja California. En Anónimo, Las perlas de Baja California. México: Departamento de Pesca.

Foley, P. and Mather, C. (2019). Ocean grabbing, terraqueous territoriality and social development. Territory, Politics, Governance, 7 (3), 297-3I5.

Garduño, R. (3.II.2008). McCarthy gestionó venta ilegal de terrenos en BCS. La Jornada.

Gerhard, P. (1956). Pearl diving in Lower California I535- I830. Pacific Historical Review, 25 (3), 239-249.

Greenpeace (2010). Cabo Cortés: destruyendo el paraíso. Greenpeace.

Guha, R. (2000). Environmentalism. A Global History. Longman World History Series.

Guha, R. (2006). How much should a person consume. Environmentalism in India and the United States. University of California Press.

Harvey, D. (2010). El enigma del capital y la crisis del capitalismo. Ediciones Akal.

Harvey, D. (20I2). Ciudades Rebeldes. Del Derecho de la Ciudad a la Revolución Urbana. Ediciones Akal.

León-Portilla, M. (2000). El ingenioso don Francisco de Ortega. Sus viajes y noticias californianas, I632-I636. En LeónPortilla, M., La California mexicana. Ensayos acerca de su historia (pp. I5 I- 188). Universidad Nacional Autónoma de México, Instituto de Investigaciones Históricas / Universidad Autónoma de Baja California.

Mathes, M. (1973). SebastiánVizcaíno y la expansión española en el océano Pacífico (I 580- I 630). Instituto de Investigaciones Históricas, Universidad Nacional Autónoma de México.

Naredo, J.M. (2006). Raíces económicas del deterioro ecológico y social. Más allá de los Dogmas. Editorial Siglo XXI.

Norgaard, R. (1996). Development Betrayed. The end of progress and a coevlutionary revisioning of the future. Routledge Press.

O’Connor, J. (1997). ¿Qué es la Historia Ecológica? ¿Por qué la Historia Ecológica? Ecología Política I4, I I5-I 3 I.

Ortega Santos, A. y Olivieri, C. (2020). Narrativas de la Colonialidad en el golfo de California (Siglos XIX-XX). En Cariño, M. y Domínguez, W. (Eds.). Extractivismo industrial y comercial del Golfo de California, I830-2019. Volumen II. Ed. Comares (en prensa). 
Sieferle, R.P. (200I). ¿Qué es la Historia Ambiental?” En González de Molina, M. y Martínez Alier, J. (Eds.): Naturaleza Transformada (pp. 3I-55). Ed. Icaria.

Uribe, T. (I 3.07.20I0). Baja California Sur, segundo lugar nacional por delitos de despojo. La Tijereta. Recuperado de: https://latijeretabcs.blogspot.com/2010/07/baja-california-sur-segundo-lugar.html

Valadés, A. (1974). Historia de la Baja California, I850-I880. Universidad Nacional Autónoma de México.

Valiente, C. (2020). Sin playas no hay paraíso. Ecología política de playas en destinos turísticos. El caso de BCS. (Tesis de Doctorado en Ciencias Sociales, DESyGLO). Universidad Autónoma de Baja California Sur, La Paz.

Vásquez, J. [1586] (1965). Real Cédula en favor de Hernando de Santotis. En Mathes, W. M. (Ed.), Californiana I. Documentos para la Historia de la demarcación comercial de la California (I583-I632), tomo I, (PP. I4-I5). Ediciones José Porrúa Turanzas.

Vizcaíno, S. [1598] (1982). Relación el primer viaje de SebastiánVizcaíno. En Portillo y Díez de Sollano, Á., Descubrimientos y exploraciones en las costas de California (pp. 339-345). Ediciones Rialp.

Worster, D. (1993). The Wealth of Nature. Environmental History and the Ecological Imagination. Oxford Univeristy Press.

Worster, D. (1998). Doing Environmental History. En Worster, D. (Ed.). The End of the Earth (pp. 289-306). Cambridge University Press. 


\section{RELACIONES INTERNACIONALES}

Revista académica cuatrimestral de publicación electrónica

Grupo de Estudios de Relaciones Internacionales (GERI)

Universidad Autónoma de Madrid, España

https://revistas.uam.es/relacionesinternacionales

ISSN 1699 - 3950

f facebook.com/RelacionesInternacionales

3. twitter.com/RRInternacional 